\title{
Advances in aeropropulsion tribology and design using five key parameters
}

\author{
L. Wedeven ${ }^{1}$, H. Chin ${ }^{2}$, W. Ogden ${ }^{2} \&$ D. Haluck ${ }^{3}$ \\ ${ }^{I}$ Wedeven Associates, Inc., USA \\ ${ }^{2}$ Pratt \& Whitney, USA \\ ${ }^{3}$ Pratt \& Whitney Rocketdyne, USA
}

\begin{abstract}
Difficult tribology problems with wear, scuffing and contact fatigue in aeropropulsion are being addressed with a systematic approach. Solutions to these problems have been found using a systematic tribology approach involving detailed failure analysis and simulation testing. The risks in advanced development have been substantially reduced by the inclusion of tribology attributes in mechanical system design. These attributes control lubrication and failure mechanisms (wear, scuffing and contact fatigue). The developmental process involves five key tribology parameters. Simulation of wear, scuffing and micro-pitting can be accomplished with the control of the five key tribology parameters: entraining velocity $\left(\mathrm{U}_{\mathrm{e}}\right)$, sliding velocity $\left(\mathrm{U}_{\mathrm{s}}\right)$, film thickness-tosurface roughness ratio $(\mathrm{h} / \sigma)$, contact stress (both global and asperity scale) and contact temperature. Specialized test machines, test specimens and test protocols that control these parameters are able to replicate the failure mechanisms experienced in service. This approach has been used to develop next generation jet engine oil formulations that are compatible with stainless steel bearing materials. Simulation testing involving oil formulations, along with bearing steel composition and heat treatments, shows what technologies have the greatest impact on performance and where future design and development efforts should be focused.
\end{abstract}

Keywords: bearing steels, adhesive wear, aeropropulsion bearings, tribology testing, systematic tribology. 


\section{Introduction}

Advances in aeropropulsion systems, such as those shown in Figure 1, are critically dependent upon a strong linkage between tribology and design. High power density and demand for reliability and long life require no tribology attribute to be left behind. A systems approach has evolved into a comprehensive process for staged technology readiness levels (TRL). The approach assures performance readiness before commitment to a new technology. It also creates an environment for innovation and a pathway to move good ideas toward maturity.
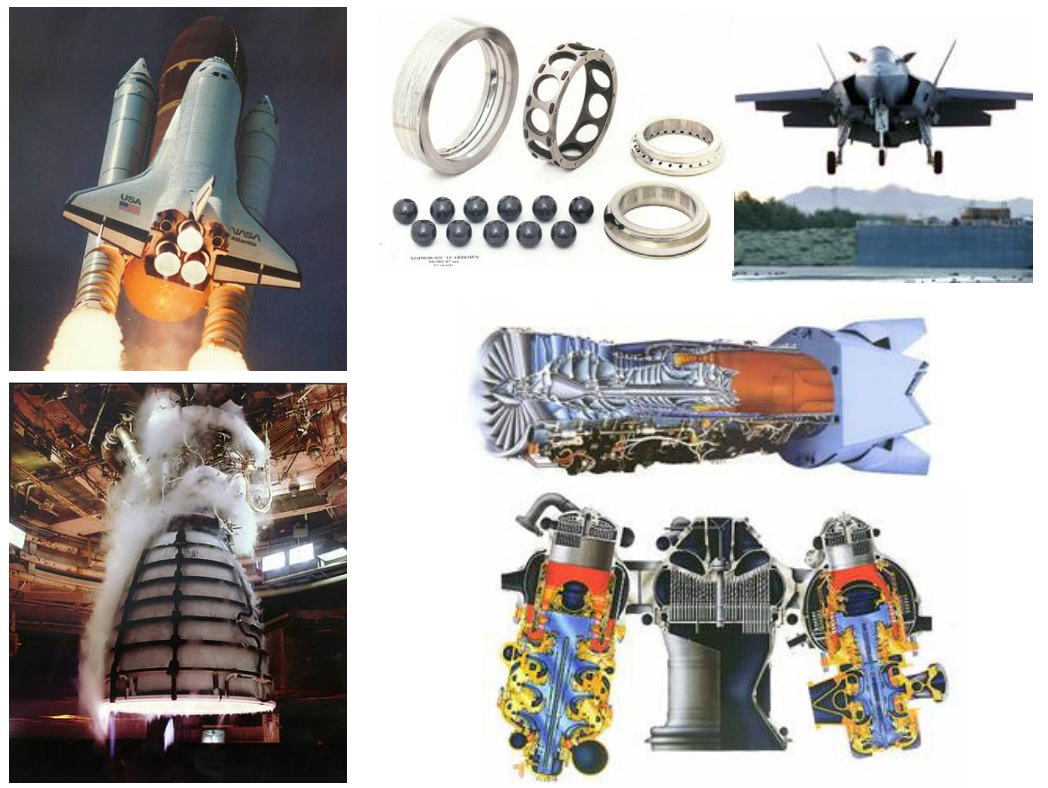

Figure 1: Aeropropulsion systems with demand for high power density.

Design of high stress lubricated contacts requires the capture of lubrication mechanisms and the avoidance of failure mechanisms. For many aeropropulsion components the margin between effective lubrication mechanisms and the onset of wear, scuffing and fatigue can be small. This paper shows how five key tribology parameters are used to define a linkage between tribology mechanisms and component design. The application of these five parameters is illustrated with an adhesive wear test protocol.

\section{Background}

In the past, development of advanced bearing materials was primarily focused on material characteristics for rolling contact fatigue resistance. Currently, the 
challenge is to develop other surface durability attributes that complement fatigue resistance attributes.

One can categorize surface deterioration into three basic modes: wear, scuffing and fatigue. The term "adhesive wear" is commonly used when failed surfaces appear to have undergone plastic flow due to local "adhesion" at the interface. The introduction of some advanced bearing materials, particularly corrosion resistant materials, has highlighted the importance of adhesive wear. Local adhesion and smearing events have been found to occur when the elastohydrodynamic (EHD) oil film thickness (h) is small relative to surface roughness height $(\sigma)$. An example of adhesive wear near the shoulder of a thrust loaded ball bearing is shown in Figure 2.

If the surface chemistry of the material does not allow the formation of surface films from reactions with the oil, adhesive wear can supersede surface deterioration due to surface initiated fatigue. In addition, material properties that affect plastic flow, like hardness and the near-surface micro-structure seem to influence the onset of adhesive wear. The mechanisms that control the ability of a surface to handle high normal and tangential stress and to recover subsequent to local damaging events are not totally understood. Testing for these mechanisms and associated surface durability attributes is essential for material and oil formulation development to assure performance in service.

It was found that surface failure by adhesive wear is initiated at microscopic sites with insufficient surface film lubrication or at sites of debris encounters. With limited chemical reactivity between lubricating oil and some corrosion resistant materials, local adhesion events, which are not able to recover, propagate into broad patches of adhesive wear damage. With sufficient sliding velocity and contact stress, adhesive wear can transition into a major scuffing event. A scuffing event is characterized by a rapid rise in friction and temperature. These tribological features, as measured with an adhesive wear test method, correlate with experience in full-scale bearing tests.

At the heart of surface durability is material compatibility with lubricating oil chemistry to form surface films. These surface films are essential for preventing local adhesion. The adhesive wear test method described below progressively

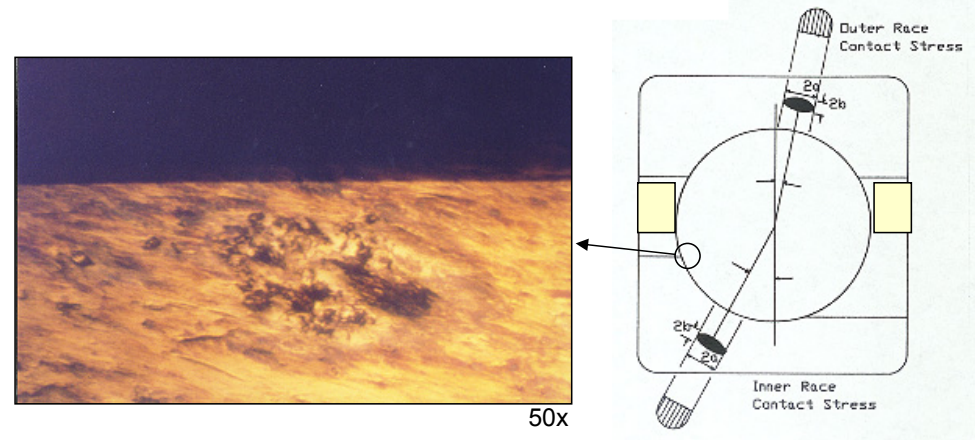

Figure 2: Adhesive wear - adhesion and plastic flow at the shoulder of a thrust loaded bearing. 
increases the degree of asperity encounter at the interface under rolling/sliding conditions. The test method invokes tribological interactions, which are measured in terms of friction (traction), gentle polishing wear of surface features, adhesive wear events and scuffing. The test protocol described below is an attempt to simulate the adhesive wear mechanisms that are believed to occur in high speed rolling element bearings.

\section{Systematic tribology}

Historical approaches for bearing, gear and lubrication development have focused on design and development of "bulk" steel properties and "bulk" oil properties for durability. Today, development is directed toward the management of the tribology attributes of the contact interface. The contact interface is considered as a "tribo-system", such that we can define four structural elements and technology regions, as shown in Figure 3.

These structural elements are: (1) hydrodynamic or elastohydrodynamic (EHD) film region; (2) surface film region; (3) near-surface region; and (4) subsurface region. These regions perform specific functions for lubrication and they provide the intrinsic properties for surface life and durability. For highly stressed lubricated contacts, durability is derived from three potentially active lubrication mechanisms which generate surface-separating films to carry the load and bear the shear at the interface. These lubrication mechanisms are: EHD, micro-EHD and surface film generation. Surface films, or boundary lubrication mechanisms, are required to preserve the integrity of contacting surfaces so that EHD and micro-EHD mechanisms can properly function. If boundary

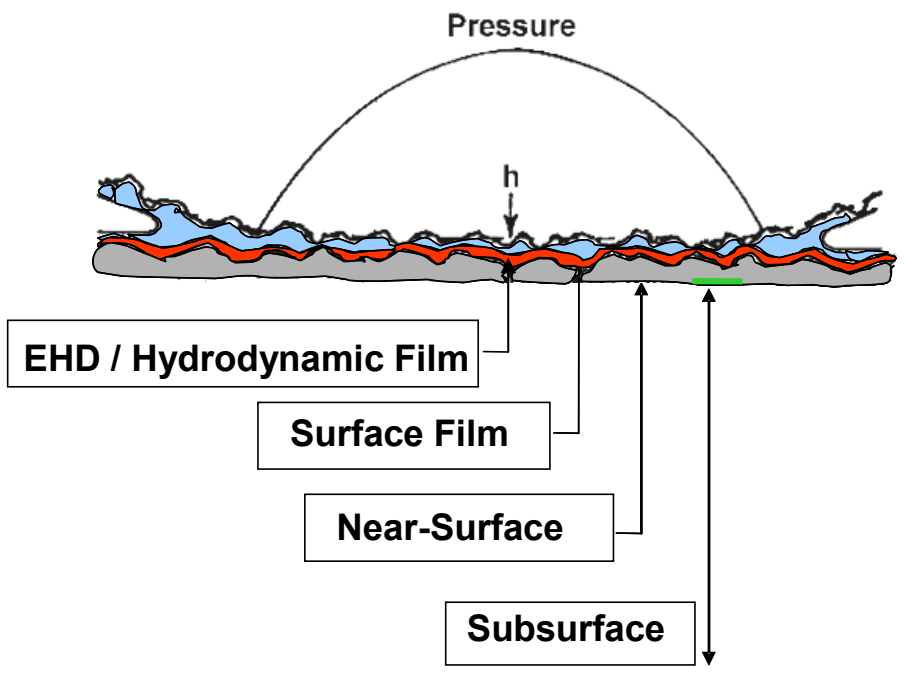

Figure 3: Structural elements of a contact. 
lubrication mechanisms are compromised, the margin for lubrication of the total tribo-system is severely compromised. Experience has clearly shown that the durability of aeropropulsion hardware is critically dependent upon all three mechanisms. Since compatible oil and material chemistry is required to create effective surface films, oil attributes and bearing material attributes are fundamentally linked.

Durability is derived from the tolerance of stresses and strains to be accommodated at asperity sites. Durability is limited by multiple mechanisms reflecting performance attributes in terms of general categories of wear, scuffing and fatigue processes. Material or surface technologies to improve one attribute are frequently at the expense of another attribute. This has been experienced in both the gearing and bearing technical communities. It is important that no attribute is left behind. To assure wear, scuffing and fatigue performance, the structural elements must be tested as a system with linkage to design through parameters that the designer can use.

\section{Five key parameters}

The challenges of bearing and gear design are to invoke lubrication mechanisms of full-film EHD, partial-film EHD and boundary film lubrication and to avoid failure mechanisms associated with wear, scuffing and fatigue. Tribology testing must capture the micro-scale lubrication and failure mechanisms controlling performance and somehow link the testing results to the macro-scale design space that the design engineer can utilize. Five key tribology parameters illustrated in Figure 4 have been found to be effective in linking tribology mechanisms in service with testing and design. The five parameters are derived from EHD theory.

The entraining velocity $\left(\mathrm{U}_{\mathrm{e}}\right)$ controls the formation of EHD film thickness through the generation of pressure in the inlet region. The sliding velocity $\left(\mathrm{U}_{\mathrm{s}}\right)$ controls the shear and heat generation with the Hertzian region. The film thickness-to-surface roughness ratio $(\mathrm{h} / \sigma)$, or Lambda, controls the degree of

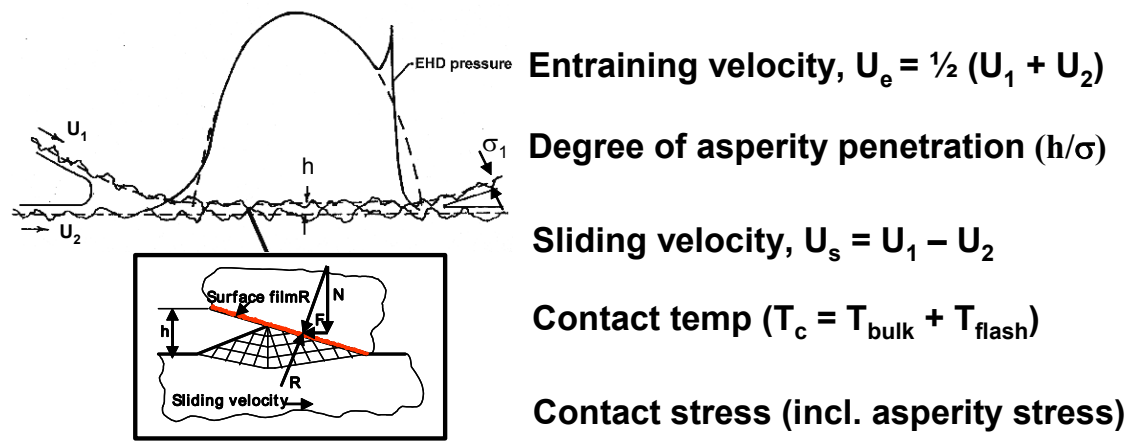

Figure 4: Five key parameters that link tribology mechanisms with design and testing. 
asperity interaction. The contact stress is considered on a global Hertzian contact scale and a local asperity scale. Adhesive wear, micro-pitting and microscuffing are generally initiated on a local scale and subsequently propagated to a global scale. Contact temperature $\left(\mathrm{T}_{\mathrm{c}}\right)$ is considered as the sum of the bulk temperature $\left(T_{b}\right)$ and the friction generated flash temperature $\left(T_{f}\right)$. The contact temperature controls reaction rate between the lubricant and surface material. It also controls the shear properties at the interface.

The five key parameters control both lubrication and failure mechanisms. They are useful for tribology design, failure analysis and testing. The ability to invoke these mechanisms in a simulation testing environment is accomplished with a patented [1] Wedeven Associates Machine (WAM) and test methodologies. The test machine is illustrated in Figure 5.

The test machine uses ball and disc test specimens with carefully prepared heat treatment, geometry, surface roughness and texture to simulate bearing or gear surfaces. Traction (friction) coefficient is measured along with bulk temperatures of the ball and disc specimens. The unique feature of WAM technology is the ability to decouple the entraining velocity, $\mathrm{U}_{\mathrm{e}}$ from the sliding velocity, $\mathrm{U}_{\mathrm{s}}$. This allows precise control of EHD film thickness, the degree of asperity interaction $(\mathrm{h} / \sigma)$ and the shear rate within the Hertzian region.

Figure 6 shows a test plot with high nitrogen stainless steel specimens. The test is operated at low $\mathrm{h} / \sigma$ with $10.16 \mathrm{~m} / \mathrm{s}$ entraining velocity $\left(\mathrm{U}_{\mathrm{e}}\right), 2.48 \mathrm{GPa}$ stress, $200 \mathrm{C}$ and $15 \%$ slip $\left(\mathrm{U}_{\mathrm{s}}=1.52 \mathrm{~m} / \mathrm{s}\right)$. The rise in traction coefficient with decreasing $U_{e}$ is attributed to local surface damage caused by metallic debris roll-over. This particular high nitrogen steel is vulnerable to adhesive wear. The

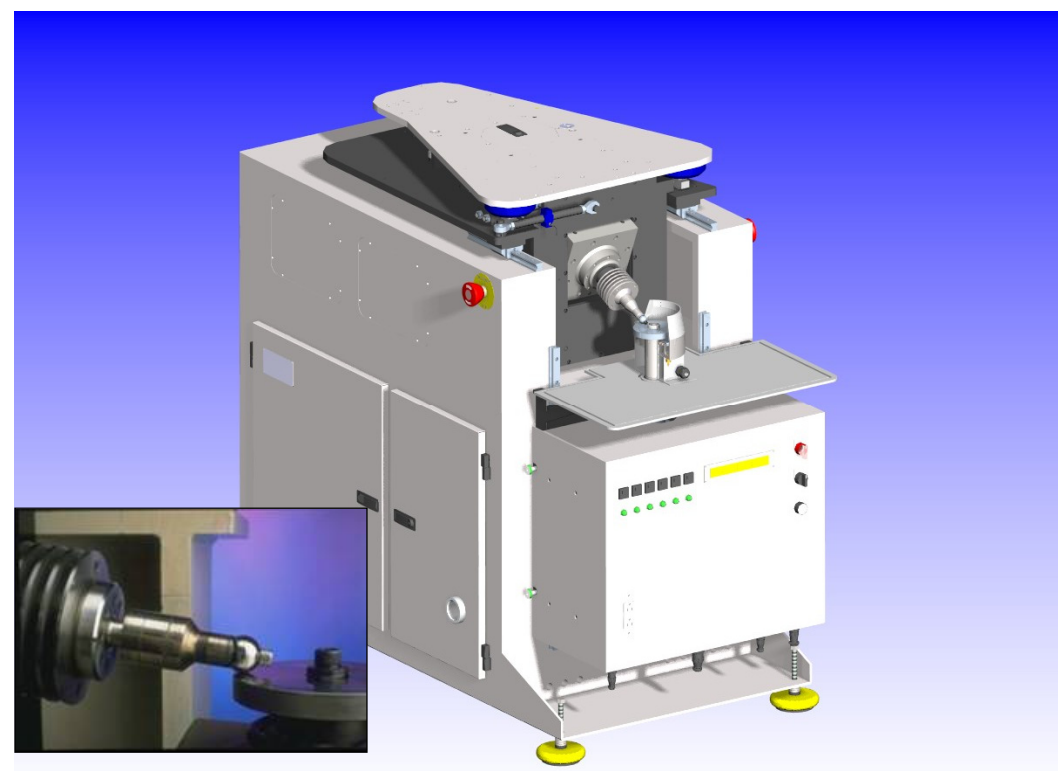

Figure 5: WAM test machine configuration. 
test was terminated before the adhesive wear damage propagated to a more massive scale (see photomicrograph in Figure 6). A test protocol was developed to screen advanced bearing steels and lubricants for adhesive wear. The test protocol is shown in Figure 7 for a ball/disc material pair of M50/M50.

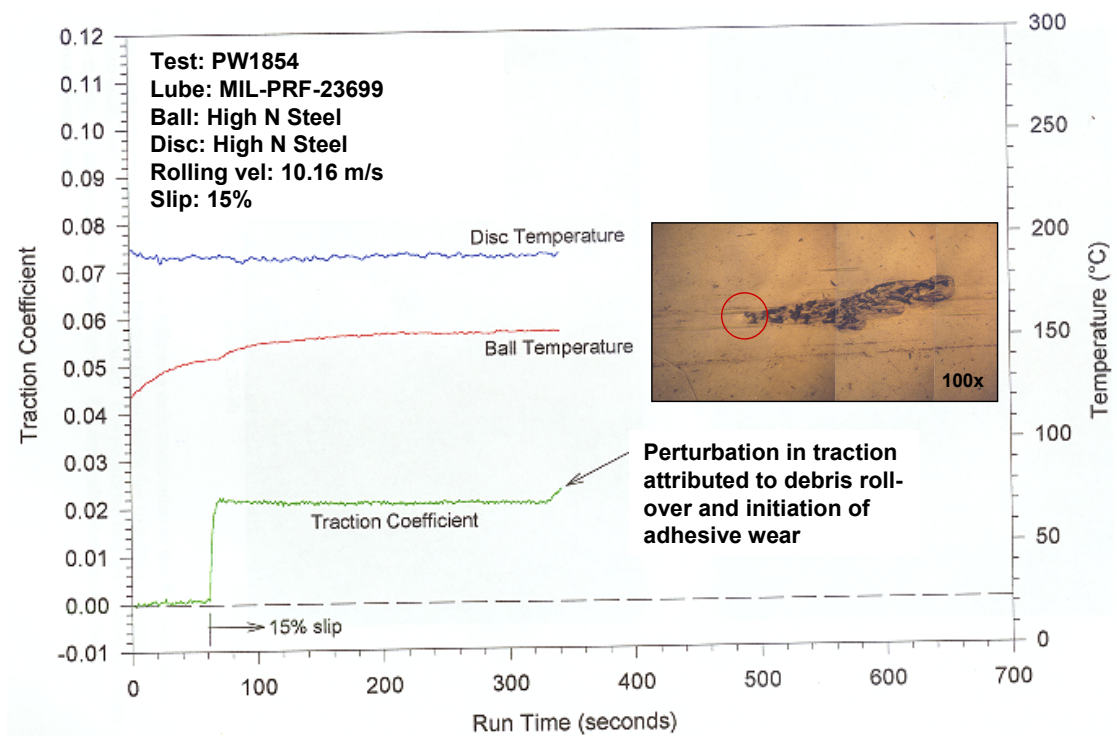

Figure 6: WAM test plot showing adhesive wear initiation at a local debris roll-over site.

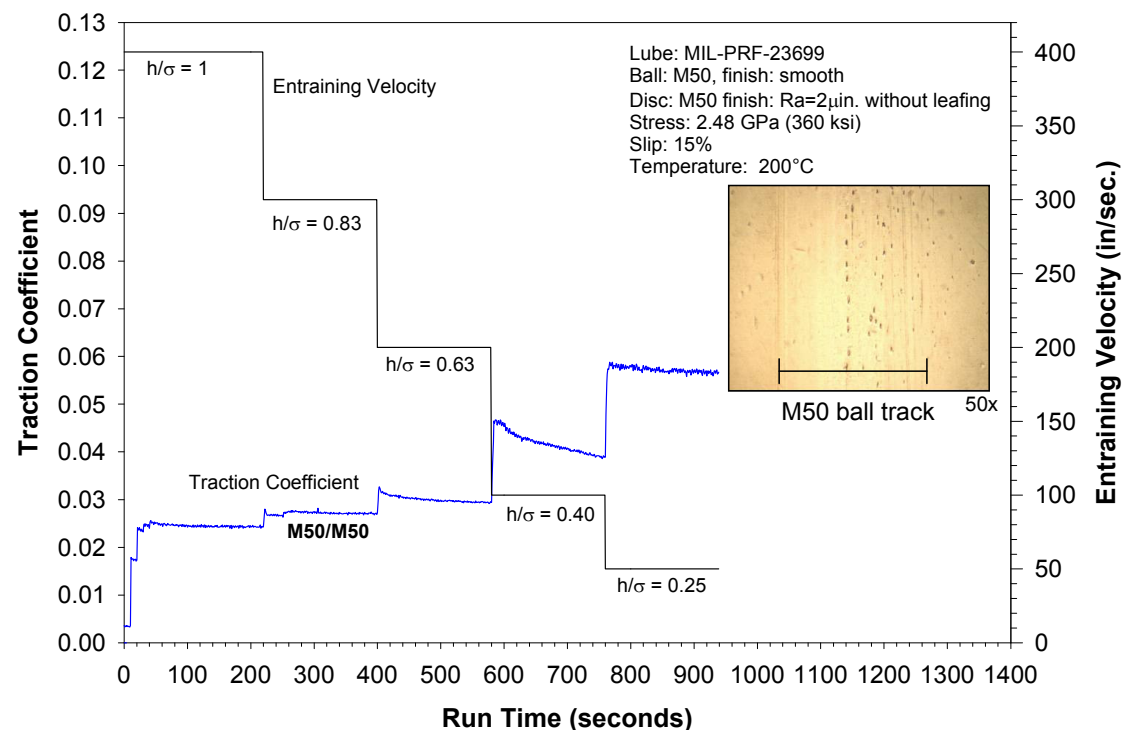

Figure 7: $\quad$ Test protocol for adhesive wear. 
The disc surface finish is precisely honed to a roughness of $0.05 \mu \mathrm{m}, \mathrm{Ra}$. The ball has an as-received lapped finish of $<0.025 \mu \mathrm{m}, \mathrm{Ra}$. The test protocol reduces $\mathrm{h} / \sigma$ in progressive steps from an initial value of 1.0 to a value of 0.25 . The test is run with a constant value of percent slip (15\%). The test is conducted at $200 \mathrm{C}$ and a maximum Hertzian stress of $2.48 \mathrm{GPa}$. At $\mathrm{h} / \sigma$ of 1.0 the traction coefficient is 0.025 . This is representative of the limiting shear strength of a full EHD film. As $U_{e}$ is reduced, the traction coefficient increases due to greater asperity interaction. A decreasing traction coefficient, like that shown for $h / \sigma$ of 0.40 , is due to minor polishing of the roughness features on the disc specimen. The adhesive wear test protocol with M50/M50 does not show any remarkable surface damage (see photomicrograph of ball specimen in Figure 7).

A repeat test using a high nitrogen stainless steel (version 1) is shown in Figure 8, along with M50. Shortly after initial operation at $\mathrm{h} / \sigma$ of 1.0 , the traction coefficient rises to approximately 0.07 due to an adhesive wear event. The local adhesion and plastic flow disturbs the integrity of the surface. While the traction coefficient shows some recovery, the surfaces and traction coefficient do not recover to their original state (see photomicrograph in Figure 8).

For those materials which pass the adhesive wear test at 15 percent slip the test is repeated at 30 percent slip and subsequently at 50\% slip. To avoid oxidation of the disc specimen at $200 \mathrm{C}$, the disc specimen is reconditioned with a light hone. The ball specimen is removed, documented and retested on the same track. The adhesive wear performance of material pairs and lubricants is judged by traction behaviour and surface deterioration features according to the criteria shown in Figure 9.

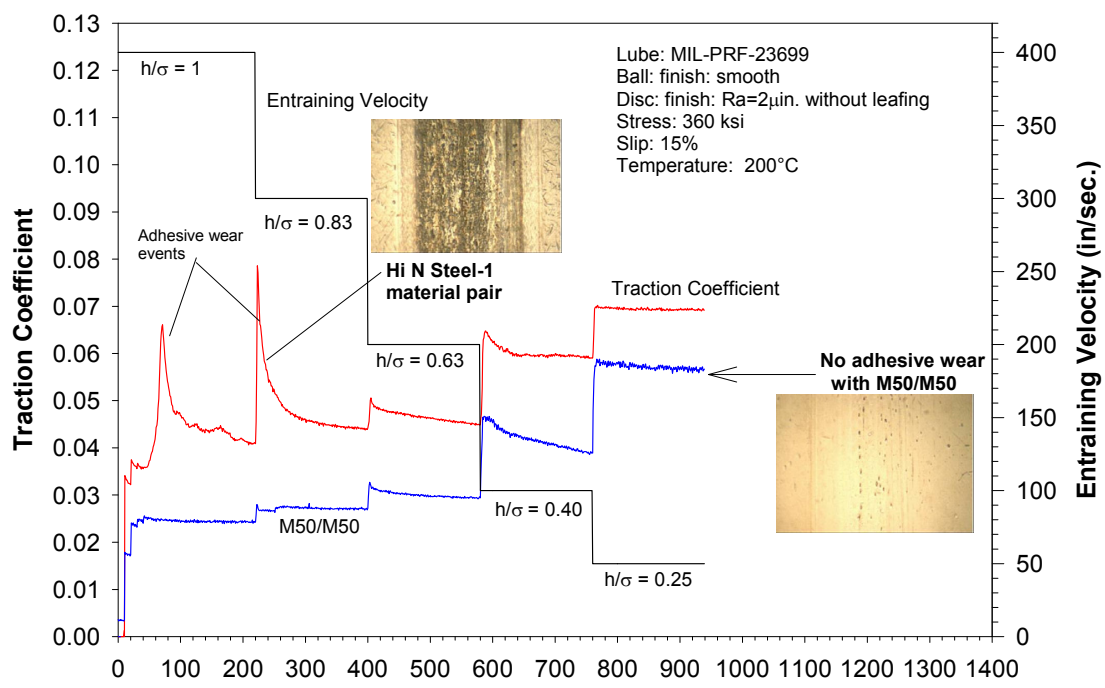

Run Time (seconds)

Figure 8: Adhesive wear test plot with high nitrogen steel (version 1) and M50/M50. 
Measured Tribology Attribute

No adhesive wear events at $15 \%$ slip.

$\underline{\text { Weighting Factor }}$

No large step increases in traction @ low $\lambda$ (15\% slip).

10

No rapid decline in traction coefficient reflecting advanced polishing wear. 4

Survives $8 \%$ slip without adhesive wear.

Survives $8 \%$ slip without major increase in traction.

Survives high slip (>15\%) without adhesive wear events:

$30 \%$ slip $=8 ; 50 \%$ slip $=17$

"Steady" traction behavior (no fluctuations due to a struggling contact). 2

Survives without a catastrophic scuff.

\section{6} 6

Figure 9: Adhesive wear performance criteria.

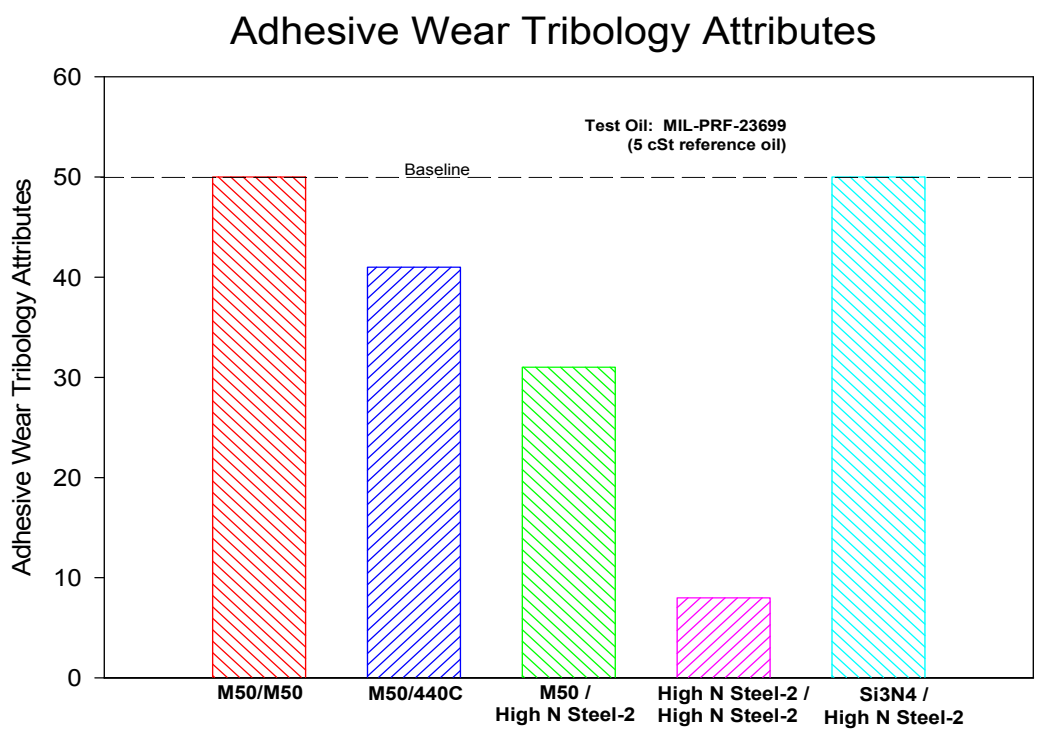

Figure 10: Adhesive wear for bearing material pairs.

Using the adhesive wear criteria in Figure 9, the test results for a combination of bearing materials are shown in Figure 10.

The mixed material pair of M50/440C shows reduced performance compared with M50/M50. The introduction of a stainless 440C material in the pair reduces adhesive wear performance. Adhesive wear performance is reduced further with high nitrogen steel (version 2). The introduction of silicon nitride with high nitrogen steel substantially improves adhesive wear.

At least some of the sensitivity of stainless steels to adhesive wear is the inherent passivation that chrome oxides have on the surface. The dominance of chrome oxides in place of iron oxides for non-corrosion resistant steels makes it difficult for anti-wear additives to sufficiently react with the steel to form protective surface films. To reduce this difficulty the bearing surfaces can be 


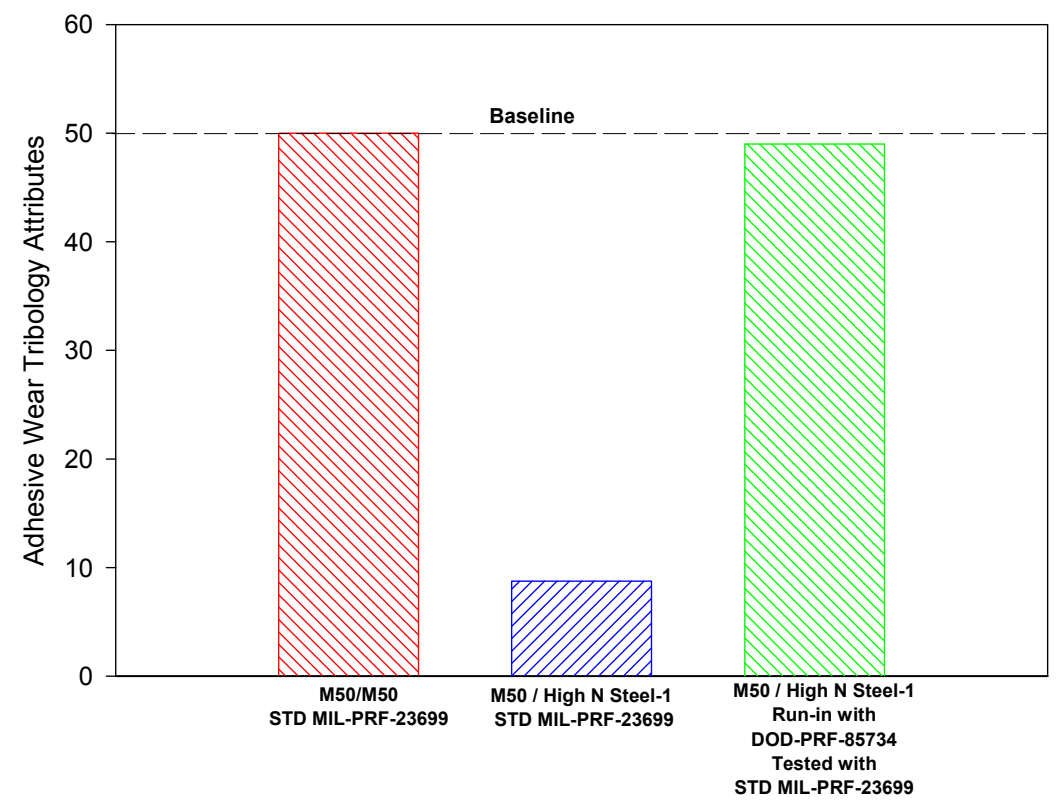

Figure 11: Effect of pre-test run-in on adhesive wear.

run-in with more aggressive additive formulations. This is shown in Figure 11. The data in Figure 11 shows that a good run-in with a more chemically active oil formulation (DOD-PRF-85734) is able to improve adhesive wear performance. This approach is limited as the run-in film is only effective so long as it is not worn off.

\section{Discussion}

The five key tribology parameters and the decoupling of $U_{e}$ and $U_{s}$ in testing provide a useful simulation testing approach for development, design and assurance of performance in service. An approach which simulates tribology mechanisms operative in service is not always intuitive. Engineers feel more comfortable with tests that operate under surface speeds and contact conditions they are familiar with. The decoupling of $\mathrm{U}_{\mathrm{e}}$ and $\mathrm{U}_{\mathrm{s}}$ provides a highly precise control of $h / \sigma$ and the shear within the contact. This allows testing methods to cover all the major lubrication and failure modes. Low $h / \sigma$ and high $U_{s}$ are required to encounter scuffing failures. Lower values of $U_{s}$ will introduce wear and micro-scuffing. The control of asperity stress and operation at low $h / \sigma$ is a way to introduce micro-pitting. The adhesive wear test protocol is only one of several test protocols used for development and qualification testing. The importance of adhesive wear testing, along with testing for other tribology 


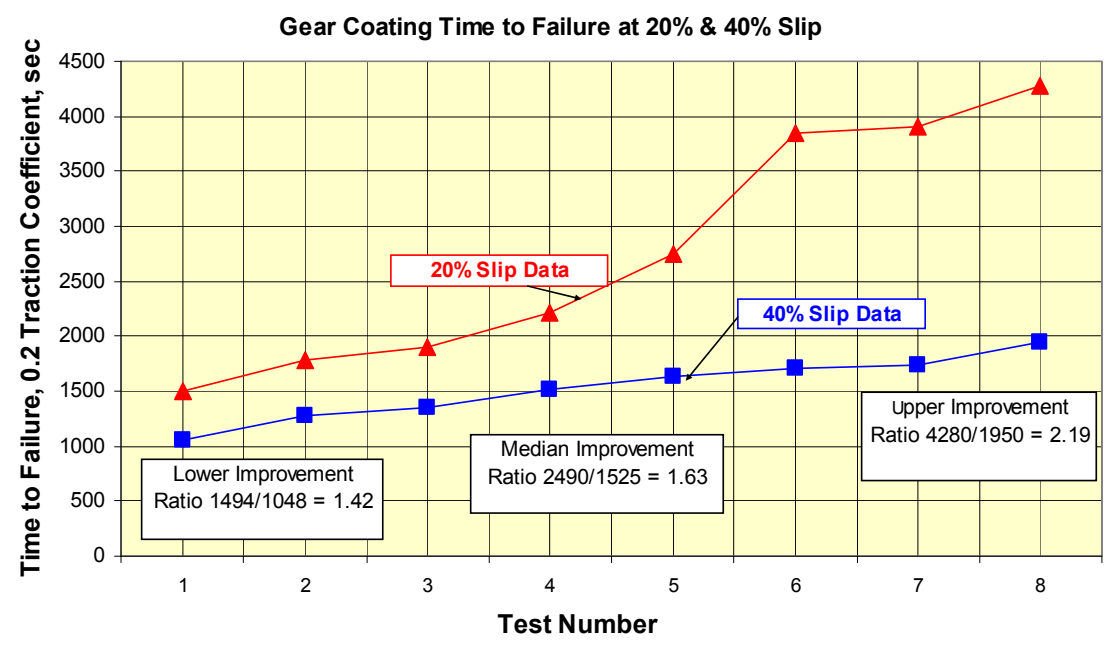

Figure 12: Cryogenic tests for rocket engine gears.

attributes, is associated with the management of performance attributes in design. For example, the adhesive wear vulnerability of high nitrogen steels can be managed. Excellent corrosion resistance and contact fatigue resistance make these materials highly desirable. Comprehensive tribology testing can map out the design space where these materials are highly effective.

Exploring the impact of key tribology parameters on performance is an excellent way to direct technical development. One example of this is the discovery of the importance of sliding velocity $\left(\mathrm{U}_{\mathrm{s}}\right)$ in gear tooth design for a specific rocket engine upgrade. Performance tests conducted at two different sliding velocities shown in Figure 12 provided critical information for redesign of the gear tooth profile that gave substantially improved performance margin.

Many aeropropulsion systems have unknown motions or operating conditions at the interface. If performance issues need to be addressed, the five key tribology parameters are used in tests to explore conditions which cause the same surface deterioration features experienced in service. The actual operating conditions are deduced from experimentation.

\section{Conclusions}

Five key tribology parameters: entraining velocity $\left(U_{e}\right)$, sliding velocity $\left(U_{s}\right)$, film thickness-to-surface roughness ratio $(\mathrm{h} / \sigma)$, contact stress and contact temperature, provide links between tribology mechanisms in service, simulation testing and design. Unique testing capability that decouples $U_{e}$ and $U_{s}$ allows precise control of high stress lubricated contact conditions to invoke all the major lubrication and failure modes. This systematic tribology approach clearly defines the adhesive wear attributes of bearing materials. Additional test methods for other attributes provide a design space for tribology performance. 
94 Tribology and Design

\section{Reference}

[1] Wedeven, L.D., "Method and Apparatus for Comprehensive Evaluation of Tribological Materials, U.S. Patent No. 5,679,883, October 21, 1997. 